\title{
A NOTE ON THE SUBADDITIVITY OF SYZYGIES
}

\author{
SABINE EL KHOURY AND HEMA SRINIVASAN
}

\begin{abstract}
Let $R=S / I$ be a graded algebra with $t_{i}$ and $T_{i}$ being the minimal and maximal shifts in the minimal graded free resolution of $R$ at degree $i$. We prove that $t_{n} \leq t_{1}+T_{n-1}$ for all $n$. As a consequence, we show that for Gorenstein algebras of codimension $h$, the subadditivity of maximal shifts $T_{n}$ in the minimal graded free resolution holds for $n \geq h-1$, i.e we show that $T_{n} \leq T_{a}+T_{n-a}$ for $n \geq h-1$.
\end{abstract}

\section{INTRODUCTION}

Let $R=S / I$ be a standard graded algebra, with $S$ a polynomial ring over a field $k$. Denote by $(\mathbb{F}, \partial)$ a minimal graded free resolution of $R$ over $S$ with $\mathbb{F}_{a}=\oplus_{j} S(-j)^{\beta_{a j}}$. For each $a$, denote by $T_{a}(\mathbb{F})$ and $t_{a}(\mathbb{F})$ the maximal and minimal shifts in the resolution $\mathbb{F}$. In other words,

$$
\begin{gathered}
t_{a}(\mathbb{F})=\min \left\{j: \beta_{a j} \neq 0\right\} \\
T_{a}(\mathbb{F})=\max \left\{j: \beta_{a j} \neq 0\right\} .
\end{gathered}
$$

$\mathbb{F}$ is said to satisfy the subadditivity condition for maximal shifts if $T_{a+b}(\mathbb{F}) \leq T_{a}(\mathbb{F})+T_{b}(\mathbb{F})$, for all $a$ and $b$. If $\mathbb{F}$ is the minimal graded free resolution of $R$ over $S$, then we write $t_{a}$ and $T_{a}$ for $t_{a}(\mathbb{F})$ and $T_{a}(\mathbb{F})$.

General bounds for $T_{n}$ in terms of $T_{1}$ were established for all $n$, by Bayer-Mumford and CavigliaSbarra in $[\mathrm{BM}]$ and $[\mathrm{CaS}]$. They prove that $T_{n} \leq\left(2 T_{1}\right)^{2^{m-2}}-1+n$ where $m=\operatorname{rank}_{k} S_{1}$. Subadditivity for maximal shifts in minimal graded free resolutions has been studied by many authors [ACI2], [EHU], [HS], [Mc]. Subadditivity for maximal shifts has been established for the highest non vanishing Tor, $\operatorname{Tor}_{p}(R, k)$. It was shown that $T_{p} \leq T_{1}+T_{p-1}$ for all graded algebras where $p=\operatorname{projdim} R$ [HS, Corollary 3], and that $T_{p} \leq T_{a}+T_{p-a}$ when $R$ is of depth zero and of dimension $\leq 1$ [EHU, Corollary 4.1]. In [ACI2], Avramov, Conca and Iyengar consider the situation when $R=S / I$ is Koszul and show that $T_{a+1}(I) \leq T_{a}+T_{1}=T_{a}+2$ for $a \leq$ height $(I)$. Further, they prove that if $S$ is Cohen-Macaulay, $\operatorname{reg}_{h+1}^{R}(k)=0$ and $\left(\begin{array}{c}a+b \\ a\end{array}\right)$ is invertible in $k$, then $T_{a+b} \leq T_{a}+T_{b}+1$. For monomial ideals, Herzog and Srinivasan show that $T_{a+1} \leq T_{a}+T_{1}$, for all $a$ [HS, Corollary 4].

It is known that the minimal graded free resolution of graded algebras may not satisfy the subadditivity for maximal shifts as shown by the counter example in [ACI2]. However, no counter examples are known for monomial ideals. In fact, it can be easily seen that the subadditivity for maximal shifts does hold for algebras generated by stable monomial or square free strongly stable monomial ideals.

In this paper, we consider the minimal and maximal degrees of the syzygies and show that for any $n$ we have $t_{n} \leq t_{1}+T_{n-1}$. As a consequence, we prove for Gorenstein algebras $S / I$ that $T_{a+b} \leq T_{a}+T_{b}$ for $a+b \geq s-1$ where $s=$ height $I=\operatorname{projdim} R$. The question remains if the subadditivity for maximal shifts holds for all Gorenstein algebras. It is particularly interesting since the simplest counter example to whether the subadditivity holds, is a codimension four almost complete intersection linked to a codimension four Gorenstein algebra. 


\section{Syzygies of Gorenstein Algebras}

Let $S=k\left[x_{1}, \ldots, x_{d}\right]$ and $I$ a homogeneous ideal in $S$. Let $\mathbb{F}$ be the minimal graded free resolution of $R=S / I$

$$
\mathbb{F}: 0 \rightarrow F_{s} \rightarrow \ldots \rightarrow F_{2} \stackrel{\partial_{2}}{\longrightarrow} F_{1} \stackrel{\partial_{1}}{\longrightarrow} S
$$

where $F_{a}=\oplus_{j} S(-j)^{\beta_{a j}}, t_{a}=\min \left\{j: \beta_{a j} \neq 0\right\}$ and $T_{a}=\max \left\{j: \beta_{a j} \neq 0\right\}$. We prove a general result for the syzygies of homogeneous algebras.

Theorem 2.1. $R=S / I$ be a graded algebras with $t_{i}$ and $T_{i}$ being the minimal and maximal shifts in the minimal graded free $S$-resolution of $R$ at degree $i$, then $t_{n} \leq t_{1}+T_{n-1}$, for all $n$.

Proof. We show the theorem by induction on $n$, where $n$ is the $n^{\text {th }}$ step of the resolution. For $n=1, T_{0}=0$ and hence $t_{1}=t_{1}$. We need to prove the theorem for $1<n \leq s$, where

$$
\mathbb{F}: 0 \rightarrow F_{s} \rightarrow \ldots \rightarrow F_{2} \stackrel{\partial_{2}}{\longrightarrow} F_{1} \stackrel{\partial_{1}}{\longrightarrow} S
$$

is the graded resolution of $R$. Let $I=\left(g_{1}, \ldots, g_{\beta_{1}}\right)$ where $\left\{g_{1}, \ldots, g_{\beta_{1}}\right\}$ is a set of minimal generators of $I$ and $F_{i}=\bigoplus_{j=1}^{\beta_{i}} S f_{i j}$ with $t_{1}=\operatorname{deg} f_{11} \leq \operatorname{deg} f_{12} \leq \cdots \leq \operatorname{deg} f_{1 \beta_{1}}=T_{1}$. Suppose $\partial_{1}\left(f_{1 j}\right)=g_{j}$ and $\partial_{n}\left(f_{n t}\right)=\sum_{i=1}^{\beta_{n-1}} r_{t i} f_{(n-1) i}$ for all $n$.

We see that $Z\left(f_{11}, f_{1 \beta_{1}}\right)=g_{1} f_{1 \beta_{1}}-g_{\beta_{1}} f_{11}$ is a non zero second syzygy. Hence, there exists an element $f_{11} * f_{1 \beta 1} \in F_{2}$ of degree $t_{1}+T_{1}$. This implies that $t_{2} \leq T_{1}+t_{1}$, and the theorem is true for $n=2$.

Assume we have constructed by induction an element $f_{11} * f_{n-2 i} \in F_{n-1}$ for all $1 \leq i \leq \beta_{n-2}$ such that

$$
\partial_{n-1}\left(f_{11} * f_{(n-2) i}\right)=\partial_{1}\left(f_{11}\right) f_{(n-2) i}-f_{11} * \partial_{n-2}\left(f_{(n-2) i}\right)
$$

and $t_{n-1} \leq t_{1}+T_{n-2}$ for $4 \leq n \leq s$. Let

$$
f_{11} * f_{(n-2) i}=\sum_{j=1}^{\beta_{n-1}} s_{i j} f_{(n-1) j} .
$$

We also have $\partial_{n-1}\left(f_{(n-1) t}\right)=\sum_{i=1}^{\beta_{n-2}} r_{t i} f_{(n-2) i}$ for all $1 \leq t \leq \beta_{n-1}$. Consider

$$
Z\left(f_{11}, f_{n-1 t}\right)=\partial_{1}\left(f_{11}\right) f_{n-1 t}-\sum_{i=1}^{\beta_{n-2}} \sum_{j=1}^{\beta_{n-1}} r_{t i} s_{i j} f_{(n-1) j}
$$


$Z\left(f_{11}, f_{n-1 t}\right)$ is a cycle in $F_{n-1}$, since

$$
\begin{aligned}
\partial_{n-1}\left(Z\left(f_{11}, f_{(n-1) t}\right)\right) & =\partial_{1}\left(f_{11}\right) \partial_{n-1}\left(f_{(n-1) t}\right)-\sum_{i=1}^{\beta_{n-2}} r_{t i} \partial_{n-1}\left(\sum_{j=1}^{\beta_{n-1}} s_{i j} f_{(n-1) j}\right) \\
& =\partial_{1}\left(f_{11}\right) \sum_{i=1}^{\beta_{n-2}} r_{t i} f_{(n-2) i}-\sum_{i=1}^{\beta_{n-2}} r_{t i} \partial_{n-1}\left(f_{11} * f_{(n-2) i}\right) \\
& =\sum_{i=1}^{\beta_{n-2}}\left[\partial_{1}\left(f_{11}\right) r_{t i} f_{(n-2) i}-\partial_{1}\left(f_{11}\right) r_{t i} f_{(n-2) i}+r_{t i} f_{11} * \partial_{n-2}\left(f_{(n-2) i}\right)\right] \\
& =\sum_{i=1}^{\beta_{n-2}} r_{t i} f_{11} * \partial_{n-2}\left(f_{(n-2) i}\right) \\
& =f_{11} * \partial_{n-2}\left(\sum_{i=1}^{\beta_{n-2}} r_{t i} f_{(n-2) i}\right) \\
& =f_{11} *\left(\partial_{n-2} \circ \partial_{n-1}\left(f_{(n-1) t}\right)\right) \\
& =f_{11} * 0 \\
& =0 .
\end{aligned}
$$

Thus, for every $t, 1 \leq t \leq \beta_{n-1}$, there exists an element $f_{11} * f_{(n-1) t} \in F_{n}$ of the same degree as $\operatorname{deg} Z\left(f_{11}, f_{(n-1) t}\right)$ that is mapped by $\partial_{n}$ onto $Z\left(f_{11}, f_{(n-1) t}\right)$. This means there is an element $f_{11} * f_{(n-1) t} \in F_{n}$ of degree $t_{1}+\operatorname{deg} f_{(n-1) t} \leq t_{1}+T_{n-1}$ such that $\partial_{n}\left(f_{11} * f_{(n-1) t}\right)=Z\left(f_{11}, f_{(n-1) t}\right)$. However, the cycles $Z\left(f_{11}, f_{n-1 t}\right)$ can be zero for all $t$ ! For that, we show that there is at least one $t$ such that one of the cycles $Z\left(f_{11}, f_{n-1 t}\right)$ is not identically zero.

Suppose that $Z\left(f_{11}, f_{n-1 t}\right)=0$ for all $t$, then $\partial_{1}\left(f_{11}\right) f_{n-1 t}=\sum_{i=1}^{\beta_{n-2}} \sum_{j=1}^{\beta_{n-1}} r_{t i} s_{i j} f_{n-1 j}$. So for all $t$, we have

$$
\sum_{i=1}^{\beta_{n-2}} \sum_{j=1}^{\beta_{n-1}} r_{t i} s_{i j}= \begin{cases}0 & j \neq t \\ \partial_{1}\left(f_{11}\right) & j=t .\end{cases}
$$

By setting $\bar{r}=\bar{r}_{n-1}=\left(r_{t i}\right)_{\beta_{n-1} \times \beta_{n-2}}$ and $\bar{s}=\left(s_{i j}\right)_{\beta_{n-2} \times \beta_{n-1}}$, we get $\bar{r} \bar{s}=\partial_{1}\left(f_{11}\right) I$ and hence the rank $\bar{r}_{n-1} \bar{s}=\beta_{n-1}$. By the exactness of the resolution, we have rank $\bar{r}_{n}+\operatorname{rank} \bar{r}_{n-1}=\beta_{n-1}$ where $\bar{r}_{n}$ is the matrix representing $\partial_{n}$. Since $\partial_{n} \neq 0$, this implies that rank $\bar{r}_{n-1}<\beta_{n-1}$ which is a contradiction.

Hence, there exists a non zero cycle in $F_{n-1}$, and we get $t_{n} \leq t_{1}+T_{n-1}$.

The following remark is an easy consequence of the duality of the minimal graded free resolution for Gorenstein algebras.

Remark 2.2. If $R=S / I$ is a Gorenstein algebra, with height $I=h$, then $T_{h} \leq T_{a}+T_{h-a}$. Since $c=T_{h}=t_{h}$, then by the duality of the minimal graded free resolution $\mathbb{F}$ we get $c-t_{h-a}=T_{a}$ for all $a=1, \cdots h-1$. This implies that $c=T_{a}+t_{h-a} \leq T_{a}+T_{h-a}$.

Theorem 2.3. For any graded Gorenstein algebra $S / I$ of codimension $h$, we have $T_{h-1} \leq T_{a}+$ $T_{h-1-a}$. Thus, $T_{n} \leq T_{a}+T_{n-a}$ for $n \geq h-1$. 
Proof. Since $S / I$ is Gorenstein, then $T_{h-1}=T_{h}-t_{1}$ and $T_{h-1-a}=T_{h}-t_{a+1}$. So, $T_{h-a-1}=$ $T_{h}-t_{a+1} \geq T_{h}-\left(t_{1}+T_{a}\right)$ by theorem 2.1. So, $T_{h-a-1} \geq T_{h}-t_{1}-T_{a}=T_{h-1}-T_{a}$ and hence $T_{h-1} \leq T_{a}+T_{h-a-1}$ as desired.

Definition 2.4. A minimal graded free resolution is said to be pure if, for all $a, F_{a}$ is generated in one degree. Hence the minimal graded free resolution is pure if it has the following shape

$$
\mathbb{F}: 0 \rightarrow S\left(-j_{s}\right)^{\beta_{s}} \rightarrow \ldots \rightarrow S\left(-j_{2}\right)^{\beta_{2}} \stackrel{\partial_{2}}{\longrightarrow} S\left(-j_{1}\right)^{\beta_{1}} \stackrel{\partial_{1}}{\longrightarrow} S
$$

Corollary 2.5. For any graded algebra $S / I$ with a pure resolution, we get $T_{n} \leq T_{1}+T_{n-1}$.

Proof. When the resolution is pure we have $t_{a}=T_{a}$ for all $a$, and hence by theorem 2.1 we get the result.

Remark 2.6. As a consequence, if $S / I$ is a Gorenstein pure algebra of codimension 4 or 5 , then its minimal graded free resolution satisfies the subadditivity condition.

Remark 2.7. As the following three examples show, the inequality can be tight even for codimension four Gorenstein algebras with pure resolutions.

Example 2.8. Let $S=k[x, y, z, w]$ and $I=\left(w^{2}, z^{2}, y^{2}-w z, w x^{2}, x^{2} z, x^{2} y+w y z, x^{3}+w x z+w y z\right)$ a height four Gorenstein ideal. We have $T_{4}=8, T_{3}=6 T_{2}=4$ and $T_{1}=3$. We get $T_{4}=2 T_{2} \leq T_{3}+T_{1}$ and $T_{3} \leq T_{2}+T_{1}$.

Example 2.9. Let $S=k[x, y, z, w]$ and $I=\left(w^{3}, w^{2} z, w^{2} y, w z^{2}, w y z-w^{2} x, w y^{2}, z^{3}+w x y, y z^{2}, x z^{2}\right.$, $\left.y^{2} z, x y z-w x^{2}, x^{2} z, y^{3}-2 w x z, x y^{2}, x^{2} y+w^{2} x, x^{3}+w x z-w^{2} x\right)$ a height four Gorenstein ideal generated in degree three and having a pure resolution. Then the minimal graded free resolution of $S / I$ satisfies the subadditivity condition. We have $T_{4}=8, T_{3}=5, T_{2}=4$ and $T_{1}=3$. Then we get $T_{4}=2 T_{2}=T_{3}+T_{1}, T_{3} \leq T_{2}+T_{1}$ and $T_{2} \leq 2 T_{1}$.

Example 2.10. Let $S=k[x, y, z, w, r, s, t]$ and $I=\left(r t, t z, t y-s t, t x, s w-s^{2}, s z, s y, s x, r w-s t-\right.$ $t^{2}, r z, y r+t w, r x-s^{2}, w^{2}+s t, w z, w y-s^{2}, w x, y z-z^{2}+r^{2}+r s+t w-s t-t^{2}, x z-r s-t w+s t+$ $\left.t^{2}, y^{2}, x y-z^{2}-r^{2}+r s+t w-s t-t^{2}, x^{2}+r s+t w-s t-t^{2}\right)$ a height seven Gorenstein ideal with $T_{7}=10, T_{6}=8, T_{5}=7, T_{4}=6, T_{3}=5, T_{2}=3$ and $T_{1}=2$. Then we get

$T_{7}=T_{6}+T_{1}=T_{5}+T_{2} \leq T_{4}+T_{3}$ and $T_{6} \leq T_{5}+T_{1}=T_{4}+T_{2} \leq 2 T_{3}$.

\section{REFERENCES}

[ACI1] L.L. Avramov, A. Conca, and S. B. Iyengar, Free resolutions over commutative Koszul algebras, Math. Res. Lett. 17 (2010), no. 2, 197-210.

[ACI2] L.L. Avramov, A. Conca, and S. B. Iyengar, Subadditivity of Syzygies of Koszul algebras, Math. Ann. 361 (2015), no. 1-2, 511-534. 1

[BM] D. Bayer, D. Mumford, What can be computed in algebraic geometry?, in Computational Algebraic Geometry and Commutative Algebra (Cortona, 1991), Sympos. Math., vol. XXXIV (Cambridge University Press, Cambridge, 1993), pp. 1-48 1

[EHU] D. Eisenbud, C. Huneke and B. Ulrich, The regularity of Tor and graded Betti numbers, Amer. J. Math. 128 (2006), no. 3, 573-605. 1

[FG] O. Fernändez-Ramos, P. Gimenez, Regularity 3 in edge ideals associated to bipartite graphs, J. Algebraic Combin. 39 (2014), no. 4, 919-937.

[CaS] G. Caviglia, E. Sbarra, Characteristic-free bounds for the Castelnuovo-Mumford regularity, Compos. Math. 141(6) (2005), 1365-1373 . 1

[HS] J. Herzog, H. Srinivasan, A note on the subadditivity problem for maximal shifts in free resolutions, Preprint 2013, arXiv:1303.6214. 1

[Mc] J. McCullough, A polynomial bound on the regularity of an ideal in terms of half the syzygies, Math. Res. Lett. 19 (2012), no. 3, 555-565 1 
Department of Mathematics, American University of Beirut, Beirut, Lebanon.

E-mail address: se24@aub.edu.1b

Department of Mathematics, University of Missouri, Columbia, Missouri-65211, USA.

E-mail address: srinivasanh@missouri.edu 